Bull. Austral. Math. Soc.

54A35, 54D20, 54E30, 54D05

VoL. 40 (1989) [83-89]

\title{
A NOTE ON CONNECTED SUBMETALINDELÖF SPACES
}

\author{
NOBUYUKI KeMOTO
}

\begin{abstract}
In this paper, we shall show that if $m$ is a natural number and for every $0 \leqq n \leqq m, 2^{\omega_{n}}<$ $2^{\omega_{n+1}}$ and $2^{\omega} \leqq \omega_{m}$ are assumed, then connected, locally Lindelöf, submetaLindelöf, normal spaces of character $\leqq 2^{\omega}$ are Lindelöf. Furthermore, we shall show that $2^{\omega}<2^{\omega}$ if and only if connected, locally Lindelöf, submetaLindelöf, normal spaces of character $\leqq 2^{\omega}$ and tightness $\leqq \omega$ are Lindelöf.
\end{abstract}

\section{INTRODUCTION}

It is known that $2^{\omega}<2^{\omega_{1}}$ implies connected, locally Lindelöf, submetaLindelöf, normal spaces of character $\leqq 2^{\omega}$ and tightness $\leqq \omega$ are Lindelöf (hence, connected, locally Lindelöf, normal Moore spaces are metrisable) see [1]. In this paper, we shall show that if $m$ is a natural number and for every $0 \leqq n \leqq m, 2^{\omega_{n}}<2^{\omega_{n+1}}$ and $2^{\omega} \leqq \omega_{m}$ are assumed, then connected, locally Lindelöf, submetaLindelöf, normal spaces of character $\leqq 2^{\omega}$ are Lindelöf. We shall also show that, in fact, the converse of Balogh's result above is also true (that is, if connected, locally Lindelöf, submetaLindelöf, normal spaces of character $\leqq 2^{\omega}$ and tightness $\leqq \omega$ are Lindelöf, then $\left.2^{\omega}<2^{\omega_{1}}\right)$.

First we present topological and set theoretical notation. All topological spaces are assumed to be regular $T_{1}$. A subset $S$ of a topological space is said to be normalised if for any $S^{\prime} \subseteq S, S^{\prime}$ and $S-S^{\prime}$ can be separated by disjoint open sets. A subset $S$ of a topological space is said to be separated if for any $x$ of $S$ there is a neighbourhood $U_{x}$ of $x$ such that $\left\{U_{x}: x \in S\right\}$ is disjoint. For a point $x$ of space $X, \chi(x, X)$ denotes the least cardinality of a neighbourhood base at $x$.

A space is Lindelöf if every open cover has a countable subcover. But in this paper, in order to consider Lindelöf properties for limit cardinals, we define $\kappa$-Lindelöfness which is different from $\kappa$-Lindelöfness in the usual sense, as follows: for a cardinal $\kappa$, a space is $\kappa$-Lindelöf if every open cover has a subcover of cardinality $<\kappa$. Thus Lindelöfness is equivalent to $\omega_{1}$-Lindelöfness, and compactness is equivelent to $\omega$-Lindelöfness.

A space is submetaLindelö (submetacompact) if every open cover has a countable family $\left\{\mathcal{U}_{n} \mid n \in \omega\right\}$ of open covers refining it such that for any $x$ in $X$ there is an $n$ in $\omega$ such that $\left|\left(\mathcal{U}_{n}\right)_{x}\right| \leqq \omega\left(<\omega\right.$, respectively), where $\left(\mathcal{U}_{n}\right)_{x}=\{U \in \mathcal{U} \mid x \in U\}$.

Received 22 September, 1988

Copyright Clearance Centre, Inc. Serial-fee code: 0004-9729/89 \$A2.00+0.00. 
A space is locally $\kappa$-Lindelöf if every point has a $\kappa$-Lindelöf neighbourhood.

For an ordinal $\alpha$ and a set $X,{ }^{\alpha} X$ denotes the set of all functions from $\alpha$ to $X$ and $X^{\alpha}$ denotes the cardinality of ${ }^{\alpha} X$. Furthermore ${ }^{<\alpha} X$ denotes the set $\bigcup_{\beta<\alpha}^{\beta} X$ and similarly $X^{<\alpha}$ denotes the cardinality of ${ }^{<\alpha} X$. A subset of an ordinal is said to be club if it is closed and unbounded in the order topology. For a function $f, f \mid A$ denotes the restriction of $f$ to $A$. For other set theoretical or topological notions or notations, see $[4,5]$ and $[6]$.

\section{RESUlts}

To prove topological results, first, we present a set theoretical assertion $\Phi$ and a topological assertion $N$.

Definition. Let $\kappa$ be an uncountable regular cardinal, $\lambda$ be a cardinal, and $S$ be a subset of $\kappa, \Phi(\kappa, \lambda, S)$ denotes the following assertion:

For every $F:{ }^{<\kappa} \lambda \rightarrow 2$, there exists a $g$ in ${ }^{\kappa} 2$ such that for any $f$ in ${ }^{\kappa} \lambda$, $\{\alpha \in S: F(f \mid \alpha)=g(\alpha)\}$ is stationary in $\kappa$.

Note that if $\Phi(\kappa, \lambda, S)$ holds, then such an $S$ must be stationary in $\kappa$.

$N(\kappa, \lambda, S)$ denotes the following assertion:

For every topological space $X$ and every normalised sequence $\left\{x_{\alpha}: \alpha \in S\right\}$ of distinct points, if for every $\alpha$ in $S, \chi\left(x_{\alpha}, X\right) \leqq \lambda$, then there is a stationary subset $S^{\prime}$ of $S$ such that $\left\{x_{\alpha}: \alpha \in S^{\prime}\right\}$ is separated.

Using the techniques of [3] and [7], we can prove the next result.

Lemma 1. $[3,7]$. Let $\kappa$ be an infinite cardinal. Then the following assertions are equivalent

(1) $2^{\kappa}<2^{\kappa^{+}}$;

(2) $\Phi\left(\kappa^{+}, 2, \kappa^{+}\right)$;

(3) $\Phi\left(\kappa^{+}, 2^{\kappa}, \kappa^{+}\right)$;

(4) $N\left(\kappa^{+}, 2^{\kappa}, \kappa^{+}\right)$.

(1) and (2) of the following result come directly from the definition. (3) of the following result is proved as in [3]. (4) is an easy consequence of (3).

LEMMA 2. Let $\kappa$ be an uncountable regular cardinal. Then the following assertions hold:

(1) If $S \subset S^{\prime} \subset \kappa$ and $\Phi(\kappa, 2, S)$ holds, then so does $\Phi\left(\kappa, 2, S^{\prime}\right)$.

(2) Let $S$ be a stationary subset of $\kappa$. Then the following are equivalent

(i) $\Phi(\kappa, 2, S)$ holds;

(ii) $\Phi(\kappa, 2, S \cap C)$ holds for any club $C$ of $\kappa$; 
(iii) $\Phi(\kappa, 2, S \cap C)$ holds for some club $C$ of $\kappa$.

(3) Let $\left\{S_{\alpha}: \alpha<\kappa\right\}$ be a family of subsets of $\kappa$. If $\Phi\left(\kappa, 2, \nabla_{\alpha<\kappa} S_{\alpha}\right)$ holds, then there is an $\alpha<\kappa$ such that $\Phi\left(\kappa, 2, S_{\alpha}\right)$ holds, where $\nabla_{\alpha<\kappa} S_{\alpha}=\{\beta<\kappa: \exists \alpha<$ $\left.\beta\left(\beta \in S_{\alpha}\right)\right\}$

(4) Let $\lambda<\kappa$ and $\left\{S_{\alpha}: \alpha<\lambda\right\}$ be a family of subsets of $\kappa$. If $\Phi\left(\kappa, 2, \underset{\alpha<\lambda}{\bigcup} S_{\alpha}\right)$ holds, then there is an $\alpha<\lambda$ such that $\Phi\left(\kappa, 2, S_{\alpha}\right)$ holds.

The next lemma can be proved as in [7].

LEMMA 3. Let $\kappa$ be an infinite cardinal, and $S$ be a subset of $\kappa^{+}$. If $\Phi\left(\kappa^{+}, 2, S\right)$ holds, then so does $N\left(\kappa^{+}, 2^{\kappa}, S\right)$.

From now on we will prove our results.

Lемма 4. Let $\kappa$ be an infinite cardinal, $S$ be a stationary subset of $\kappa^{+}, X$ be a submetalindelöf normal space of character $\leqq 2^{\kappa}$, and $Y$ be a subset $\left\{x_{\alpha}: \alpha \in S\right\}$ of distinct points of $X$ such that for any point $x$ of $X$ there is a neighbourhood $U_{x}$ of $x$ with $\left|U_{x} \cap Y\right| \leqq \kappa$. Assume $\Phi\left(\kappa^{+}, 2, S\right)$. Then there is a stationary subset $S^{\prime}$ of $S$ such that $\Phi\left(\kappa^{+}, 2, S^{\prime}\right)$ holds and $\left\{x_{\alpha}: \alpha \in S^{\prime}\right\}$ is closed discrete in $X$.

Proof: Take $U_{x}$ for each point $x$ in $X$ as above. Then $\mathcal{U}=\left\{U_{x}: x \in X\right\}$ is an open cover of $X$. By submetaLindelöfness, there is a sequence $\left\{\mathcal{U}_{n}: n \in \omega\right\}$ of open covers refiniug $\mathcal{U}$ such that for any point $x$ in $X$, there is an $n(x) \in \omega$ with $\left|\left(\mathcal{U}_{n(x)}\right)_{x}\right| \leqq \omega$. Let $Y_{n}$ be the subset $\left\{x_{\alpha} \in Y: n\left(x_{\alpha}\right)=n\right\}$ of $Y$, and $S_{n}$ be the subset $\left\{\alpha \in S: x_{\alpha} \in Y_{n}\right\}$ of $S$. Then $Y=\bigcup_{n \in \omega} Y_{n}$ and $S=\bigcup_{n \in \omega} S_{n} . \mathrm{By} \Phi\left(\kappa^{+}, 2, S\right)$ and (4) of Lemma 2 , there is an $n \in \omega$ such that $\Phi\left(\kappa^{+}, 2, S_{n}\right)$. Define an equivalence relation $\simeq$ on $S_{n}$ as follows: for $\alpha$ and $\alpha^{\prime}$ in $S_{n}, \alpha \simeq \alpha^{\prime}$ if and only if there is a finite sequence $U_{0}, \ldots, U_{i}$ of elements of $\mathcal{U}_{n}$ such that $x_{\alpha} \in U_{0}, x_{\alpha^{\prime}} \in U_{i}$ and $U_{j} \cap U_{j+1} \bigcap Y_{n} \neq 0$ for $j \in i$.

Since $\mathcal{U}_{n}$ is a refinement of $\mathcal{U}$, each equivalence class of $\simeq$ is of cardinality $\leqq \kappa$. Let $\left\{S_{n \gamma}: \gamma \in I\right\}$ enumerate these equivalence classes. For $\gamma$ in $\Gamma$, enumerate $S_{n \gamma}=\left\{\alpha_{\gamma \beta}: \beta<\kappa\right\}$ in type $\kappa$. For $\beta<\kappa$, let $T_{\beta}$ be the set $\left\{\alpha_{\gamma \beta}: \gamma \in \Gamma\right\}$. Then $\left\{\left\{x_{\alpha}: \alpha \in T_{\beta}\right\}: \beta<\kappa\right\}$ is a partition of $Y_{n}$; and since $\mathcal{U}_{n}$ is an open cover, $\left\{x_{\alpha}: \alpha \in T_{\beta}\right\}$ is closed discrete in $X$ for each $\beta<\kappa$. Then by (4) of Lemma 2, there is a $\beta<\kappa$ such that $\Phi\left(\kappa^{+}, 2, T_{\beta}\right)$. Hence this $T_{\beta}$ is the desired $S^{\prime}$.

From now on, we assume that $\kappa$ is an infinite cardinal.

LEMMA 5. $\left[2^{\kappa}<2^{\kappa^{+}}\right]$. Let $X$ be a submetaLindelöf, normal space of character $\leqq 2^{\kappa}$. Then the closure of $\kappa^{+}$-Lindelöf subspaces of $X$ are $\kappa^{+}$-Lindelöf. 
Proof: Suppose, on the contrary, that there is a $\kappa^{+}$-Lindelö subspace $Z$ such that $\operatorname{cl} Z$ is not $\kappa^{+}$-Lindelöf.

FACT. cl $Z$ contains a closed discrete subspace $A$ of cardinality $\kappa^{+}$.

Proof of the FACT. Let $\mathcal{U}$ be an open cover of $\operatorname{cl} Z$ with no subcover of cardinality $\leqq \kappa$. Since $X$ is submetaLindelöf, so is $\mathrm{dl} Z$. Hence there is a sequence $\left\{\mathcal{U}_{n}: n \in \omega\right\}$ of open covers of $\mathrm{cl} Z$ each refining $\mathcal{U}$ such that for any point $x$ in $\operatorname{cl} Z$ there is an $n(x) \in \omega$ such that $\left|\left(\mathcal{U}_{n(x)}\right)_{x}\right| \leqq \omega$. For $n \in \omega$, let $Z_{n}$ be the subspace $\{x \in \operatorname{cl} Z: n(x)=n\}$. By the axiom of choice, take a maximal subset $A_{n}$ of $Z_{n}$ such that no member of $\mathcal{U}_{n}$ contains two elements of $A_{n}$. Since the $\mathcal{U}_{n}$ 's are open covers of the closed subspace $\mathrm{cl} Z$, the $A_{n}$ 's are closed discrete in $X$. Let. $\mathcal{U}_{n}^{\prime}$ be the subfamily $\left\{U \in \mathcal{U}_{n}: U \cap A_{n} \neq 0\right\}$ of $\mathcal{U}_{n}$. Then by maximality, $\mathcal{U}_{n}^{\prime}$ covers $Z_{n}$ for each $n \in \omega$. Thus $\mathcal{U}^{\prime}=\bigcup_{n \in \omega} \mathcal{U}_{n}^{\prime}$ is an open cover of $\mathrm{cl} Z$ which refines $\mathcal{U}$. Since $\mathcal{U}$ has no subcover of cardinality $\leqq \kappa$, there is an $n \in \omega$ such that the cardinality of $\mathcal{U}_{n}^{\prime}$ is greater than $\kappa$. But since for any point $x$ in $A_{n},\left|\left(\mathcal{U}_{n}\right)_{x}\right| \leqq \dot{\omega}$, it follows that $\kappa<\left|A_{n}\right|$. Take a subset $A$ of $A_{n}$ of cardinality $\kappa^{+}$. This $A$ is as desired. The proof of the fact is complete.

By the fact, let $\left\{x_{\alpha}: \alpha<\kappa^{+}\right\}$be a closed discrete subset of $\operatorname{cl} Z$. By $2^{\kappa}<2^{\kappa^{+}}$ and Lemma 1 , there is a stationary subset $S$ of $\kappa^{+}$such that $\left\{x_{\alpha}: \alpha \in S\right\}$ is separated. Then by normality, take a discrete family $\left\{U_{\alpha}: \alpha \in S\right\}$ of open sets such that $x_{\alpha} \in U_{\alpha}$ for each $\alpha \in S$. Then $\left\{U_{\alpha} \cap Z: \alpha \in S\right\}$ is a discrete family of open sets of $Z$ of cardinality $\kappa^{+}$. This contradicts the $\kappa^{+}$-Lindelöness of $Z$.

TheOREM 6. $\left[2^{\kappa}<2^{\kappa^{+}}\right]$Let $X$ be a connected, $\kappa^{++}$-Lindelöf, locally Lindelöf, submetaLindelöf, normal space of character $\leqq 2^{\kappa}$. Then $X$ is $\kappa^{+}$-Lindelöf.

Proof: Assume that $X$ is $\kappa^{++}$-Lindelö but not $\kappa^{+}$-Lindelöf. Then there is an open cover $U=\left\{U_{\alpha}: \alpha<\kappa^{+}\right\}$of $X$ such that for any $\alpha<\kappa^{+}, \operatorname{cl} U_{\alpha}$ is Lindelöf and $U_{\alpha}-\bigcup_{\beta<\alpha} U_{\beta} \neq 0$. By connectedness, take a point $x_{\alpha}$ of $\partial\left(\bigcup_{\beta<\alpha} U_{\beta}\right)$ for each $\alpha<\kappa^{+}$. For $\alpha<\kappa^{+}$, take $g(\alpha) \geq \alpha$ such that $x_{\alpha} \in U_{g(\alpha)}$. Then $C=\left\{\alpha<\kappa^{+}: g^{\prime \prime} \alpha \subset \alpha\right\}$ is a club set in $\kappa^{+}$, and the points of $Y=\left\{x_{\alpha}: \alpha \in C\right\}$ are all distinct. Then by Lemma 1 and 2) of Lemma $2, \Phi\left(\kappa^{+}, 2, C\right)$ holds. Since $\mathcal{U}$ is an open cover of $X$ such that each member of $\mathcal{U}$ intersects $Y$ in at most $\kappa$-many points, by Lemma 4 there is a stationary subset $S$ of $C$ such that $\left\{x_{\alpha}: \alpha \in S\right\}$ is closed discrete and $\Phi\left(\kappa^{+}, 2, S\right)$ holds. Hence by Lemma 3 , there is a stationary subset $S_{0}$ of $S$ such that $\left\{x_{\alpha}: \alpha \in S_{0}\right\}$ is separated. Using normality, take a discrete open family $\left\{V_{\alpha}: \alpha \in S_{0}\right\}$ such that $x_{\alpha} \in V_{\alpha}$ for each $\alpha$ in $S_{0}$. Since $x_{\alpha}$ is in $\partial\left(\bigcup_{\beta<\alpha} U_{\beta}\right)$ for any $\alpha \in S_{0}$, there is an $f(\alpha)<\alpha$ such that $U_{f(\alpha)} \cap V_{\alpha}$ is non-empty. By the pressing down lemma, there are a 
stationary subset $S_{1}$ of $S_{0}$ and a $\beta<\kappa^{+}$such that $f(\alpha)=\beta$ for every $\alpha$ in $S_{1}$. Then $\left\{V_{\alpha} \cap \operatorname{cl} U_{\beta}: \alpha \in S_{0}\right\}$ is a discrete open family of cardinality $\kappa^{+}$. This contradicts the Lindelöfness of $\mathrm{cl} U_{\beta}$.

Theorem 7. Let $m$ be a natural number. Assume that for each $n, 0 \leqq n \leqq m$, $2^{\omega_{n}}<2^{\omega_{n+1}}$ holds. Let $X$ be a connected, locally Lindelöf, submetaLindelöf, normal space of character $\leqq 2^{\omega}$ and tightness $\leqq \omega_{m}$. Then $X$ is Lindelöf.

ProOF: Let $\mathcal{U}$ be an open cover of $X$ whose elements have non-empty Lindelöf closures. By Lemma 5 and $2^{\omega_{m}}<2^{\omega_{m+1}}$, the closures of $\omega_{m+1}$-Lindelof subspaces are $\omega_{m+1}$-Lindelöf. Hence by induction on $\omega_{m+1}$, take a sequence $\left\{\mathcal{U}_{\beta}: \beta \in \omega_{m+1}\right\} \subset$ $[\mathcal{U}] \leqq \omega_{m}$. such that $\mathcal{U}_{\beta}$ is non-empty and $\mathrm{cl}\left(\bigcup \mathcal{U}_{\beta}\right) \subseteq \bigcup \mathcal{U}_{\beta+1}$ for each $\beta$ in $\omega_{m+1}$. Let $\mathcal{U}^{\prime}$ be the family $\bigcup_{\beta<\omega_{m+1}} \mathcal{U}_{\beta}$. Then $\cup \mathcal{U}^{\prime}$ is clopen in $X$. Indeed, take a point $x$ in $\mathrm{cl}\left(\cup \mathcal{U}^{\prime}\right)$. Since the tightness is $\leqq \omega_{m}$, take an $\omega_{m}$-sequence $A=\left\{x_{\alpha}: \alpha<\omega_{m}\right\}$ of points in $\bigcup \mathcal{U}^{\prime}$ such that $x \in \operatorname{cl} A$. For $\alpha<\omega_{m}$, take a $\beta(\alpha)<\omega_{m+1}$ such that $x_{\alpha} \in \bigcup \mathcal{U}_{\beta(\alpha)}$. Let $\beta=\sup \left\{\beta(\alpha): \alpha<\omega_{m}\right\}<\omega_{m+1}$. Then $A \subseteq \bigcup \mathcal{U}_{\beta}$. Hence, $x \in \operatorname{cl}\left(\bigcup \mathcal{U}_{\beta}\right) \subset \cup \mathcal{U}^{\prime}$. This implies $\cup \mathcal{U}^{\prime}$ is a closed supspace of $X$. It is evident that $\cup \mathcal{U}^{\prime}$ is open. Hence it is clopen in $X$. Then by the connectedness of $X, \cup \mathcal{U}^{\prime}=X$. Since $\left|\mathcal{U}^{\prime}\right| \leqq \omega_{m+1}$, the above argument implies that $X$ is $\omega_{m+2}$-Lindelö. Therefore by Theorem $6, X$ is $\omega_{m+1}$-Lindelöf. Then by a finite number of applications of Theorem 6 , it follows that $X$ is $\omega_{1}$-Lindelof (that is, $X$ is Lindelöf). The proof is complete $\square$

Corollany 8. Let $m$ be a natural number. Assume that for each $n, 0 \leqq n \leqq m$, $2^{\omega_{n}}<2^{\omega_{n+1}}$ holds. Let $X$ be a locally connected, locally Lindelöf, submetaLindelöf, normal space of character $\leqq 2^{\omega}$ and tightness $\leqq \omega_{m}$. Then $X$ is a free union of Lindelöf subspaces (hence it is strongly paracompact).

Proof: Apply the above theorem to each connected component.

Corollary 9. Assume that for each $n, 0 \leqq n \leqq m, 2^{\omega_{n}}<2^{\omega_{n+1}}$ holds and $2^{\omega} \leqq$ $\omega_{m}$. Then connected (locally connected), locally Lindelöf, submetaLindelöf, normal spaces of character $\leqq 2^{\omega}$ are Lindelöf (a free union of Lindelöf subspaces, respectively).

Proof: Since the tightness is not greater than the character which is $2^{\omega} \leqq \omega_{m}$, we can apply Theorem 7 (Corollary 8 , respectively).

The next result was announced in $[\mathbf{1}]$.

Corollary 10. $\left[C H+2^{\omega_{1}}<2^{\omega_{2}}\right]$ Connected (locally connected) locally Lindelöf, submetaLindelöf, normal spaces of character $\leqq 2^{\omega}$ are Lindelöf (a free union of Lindelöf subspaces, respectively).

The next result was proved in [1]. 
Corollary 11. If $2^{\omega}<2^{\omega_{1}}$ holds, then connected (locally connected), locally Lindelöf, submetaLindelöf, normal spaces of character $\leqq 2^{\omega}$ and tightness $\leqq \omega$ are Lindelöf (a free union of Lindelöf subspaces, respectively).

Next we shall show that, in fact, the converse of Corollary 11 is also true.

THEOREM 12. The following assertions are equivalent:

(1) $2^{\omega}<2^{\omega_{1}}$ holds;

(2) connected, locally Lindelöf, submetaLindelö, normal spaces of character $\leqq 2^{\omega}$ and tightness $\leqq \omega$ are Lindelöf;

(3) conuected, locally Lindelö, submetacompact, normal spaces of character (weight) $\leqq 2^{\omega}$ and tightness $\leqq \omega$ are Lindelöf.

Proof: $(1) \rightarrow(2)$ : by Corollary 11.

$(2) \rightarrow(3)$ : evident.

$(3) \rightarrow(1)$ : assume $2^{\omega}=2^{\omega_{1}}$. We shall show that the negation of (3) holds. Then there is a collection of $\omega_{1}$-many free ultrafilters on $\omega$, say $\left\{x_{\alpha}: \alpha<\omega_{1}\right\}$, such that for any subset $D$ of $\omega_{1}$ there is a subset $U$ of $\omega$ such that $U \in x_{\alpha}$ for every $\alpha \in D$ and $\omega-U \in x_{\alpha}$ for every $\alpha \in \omega_{1}-D$, by [2].

Let $R$ be the real line. Since $R$ is normal and $\omega$ is closed in $P$ (hence $\omega$ is $C^{\star}$-embedded in $\left.\mathbf{R}\right), \beta \omega=\operatorname{cl}_{\beta \mathbf{R}} \omega \subset \beta R$ is valid. Here $\beta Y$ denotes the Stone-Čech compactification of a Tychonoff space $Y$. Let $X$ be $\mathbb{R} \bigcup\left\{x_{\alpha}: \alpha<\omega_{1}\right\}$. Equip $X$ with the subspace topology on $\beta \mathrm{R}$. We shall show that this $X$ has the desired properties.

Since $\boldsymbol{P}$ is connected and dense in $X, X$ is connected.

To show the normality of $X$, it is enough to show that the subspace $X-\mathrm{R}$ is normalised in $X$. Let $D$ be a subset of $\omega_{1}$, and $U$ be a subset of $\omega$ as above. Let $W$ be the set $\bigcup\{(n-1 / 2, n+1 / 2): n \in U\}$, and $W^{\prime}$ be an open set of $X$ such that $W^{\prime} \cap \mathbf{R}=$ $W$. Then it is not hard to show that $\left\{x_{\alpha}: \alpha \in D\right\} \subseteq W^{\prime}$ and $\operatorname{cl} W^{\prime} \cap\left\{x_{\alpha}: \alpha \in\right.$ $\left.\omega_{1}-D\right\}=0$. Hence $X$ is normal. This argument implies $\left\{x_{\alpha}: \alpha<\omega_{1}\right\}$ is closed discrete in $X$. Therefore $X$ is not lindelöf.

Since points of $\mathbf{R}$ have compact neighbourhoods in $X$, to show the local Lindelöfness of $X$ we must show that points of $X-R$ have closed Lindelö neighbourhoods in $X$. Take an open neighbourhood $U$ of $x_{\alpha}$ such that $\operatorname{cl} U \cap\left\{x_{\alpha}: \alpha<\omega_{1}\right\}=\left\{x_{\alpha}\right\}$. Since $\mathbf{P}$ is hereditarily Lindelöf, it follows that $\mathrm{cl} U$ is Lindelö. Thus $X$ is locally Linclelöf.

To show $X$ is submetacompact, let $U$ be an open cover of $X$. For $\alpha<\omega_{1}$, fix an $U_{\alpha} \in \mathcal{U}$ such that $x_{\alpha} \in U_{\alpha}$. For $n \in \omega$ and $\alpha \in \omega_{1}$, let $U_{\alpha n}$ be the open set $U_{\alpha}-\left([-n, n] \cup\left\{x_{\beta}: \beta \neq \alpha, \quad \beta<\omega_{1}\right\}\right)$ of $X$. By the paracompaciness of $\mathbf{R}$, take a lucally finite open family $\mathcal{V}$ refining $\mathcal{U}$ such that $\bigcup \mathcal{V}=\mathbf{R}$. Then $\left\{\mathcal{U}_{n}: n \in \omega\right\}$, where $U_{n}=\mathcal{V} \cup\left\{U_{\alpha n}: \alpha<\omega_{1}\right\}$, shows the submetacompactness of $X$. 
Since $R$ has a countable basis, the weight of $X$ is not greater than $2^{\omega}$.

Finally since $\mathrm{P}$ is hereditarily separable, the tightness of $X$ is $\omega$. The proof is complete.

\section{REFERENCES}

[1] Z. Balogh, 'Paracompactness in locally Lindelö spaces.', Canad. J. Math. 38 (1986), 719-727.

[2] A. Charlesworth, R. Hodel and F.D. Tall, 'On a theorem of Jones and Heath concerning discrete subspaces', Colloq. Math. 34 (1975), 33-37.

[3] K.J. Devlin, S. Shelah, 'A weak version of $\diamond$ which follows $2^{\mathrm{N}_{0}}<2^{\mathrm{K}_{1}}$, Israel J. Math. 29 (1978), $239-247$.

[4] R. Engelking, Outline of General Topology (Polish Scientific Publishers, Warszawa, 1965).

[5] T. Jech, Set Theory (Academic Press, 1978).

[6] K. Kunen, Set Theory (North-Holland, 1980).

[7] A.D. Taylor, 'Diamond principles, ideals and the normal Moore problem', Canad. J. Math. 33 (1981), 282-296.

Department of Mathematics,

College of Education,

Ryukyu University,

Nishilıra-Cho, Okinawa,

Japan.

\author{
Current address: \\ Depariment of Mathematics, \\ Faculty of Education, \\ Oita University, \\ Dannoharu, Oita, 870-11 \\ Japan.
}

